\title{
Synthesis and evaluation of spherical quasi plane wave regions for antenna pattern measurements
}

\author{
Hald, Jørgen
}

Published in:

Antennas and Propagation Society International Symposium

Publication date:

1981

Document Version

Publisher's PDF, also known as Version of record

Link back to DTU Orbit

Citation (APA):

Hald, J. (1981). Synthesis and evaluation of spherical quasi plane wave regions for antenna pattern

measurements. In Antennas and Propagation Society International Symposium (pp. 573-576). IEEE.

\section{General rights}

Copyright and moral rights for the publications made accessible in the public portal are retained by the authors and/or other copyright owners and it is a condition of accessing publications that users recognise and abide by the legal requirements associated with these rights.

- Users may download and print one copy of any publication from the public portal for the purpose of private study or research.

- You may not further distribute the material or use it for any profit-making activity or commercial gain

- You may freely distribute the URL identifying the publication in the public portal

If you believe that this document breaches copyright please contact us providing details, and we will remove access to the work immediately and investigate your claim 
SYNTHESIS AND EVALUATION OF SPHERICAL QUASI PLANE WAVE

REGIONS FOR ANTENNA PATTERN MEASUREMENTS

\author{
Jørgen Hald \\ Institute for Numerical Analysis \\ The Technical University of Denmark \\ DK-2800 Lyngby Denmark
}

By reciprocity the far-field pattern of an antenna is its response to an incident plane wave as a function of the direction of plane wave incidence. Thus the various types of test ranges may be regarded as different ways of establishing a quas $i$ plane wave in a volume containing the antenna under test, [1]. This again leads to the idea, that different ranges may be evaluated and compared through the quality of the synthesized or generated quasi plane waves, [1], [2]. In connection with this two major problems arise:

I) To choose a convenient figure of merit for the quality of the quasi plane wave.

II) To evaluate the measurement error arising from imperfection in the plane wave.

Two different ways of evaluating the quality of the plane wave zone shall be described. The second method is deduced from II) above, and it seems to be very efficient for the synthesis problem, which has been considered by several authers recently, [2], [3].

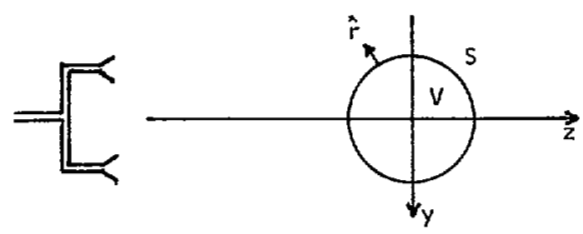

The figure illustrates the geometry. A spherical surface $S$ enclosing the quasi plane wave zone $V$ is centered in the origin. All sources of the quasi plane wave $(\underline{E}, \underline{H})$ must be outside $S$. Now, this wave is written as a sum of an ideal plane wave $\left(\mathrm{E}^{\mathrm{P}}, \mathrm{H}^{\mathrm{P}}\right)$ propagating in the positive $z$-direction and an error field $\left(E^{\varepsilon}, \underline{H}^{\varepsilon}\right)$. $\underline{E}^{P}$ is purely $x-$ polarized and equaTs the $x$-component of $E$ in the origin. Inside $S$ the error field (EE, $\underline{H}^{E}$ ) is expanded in spherical waves:

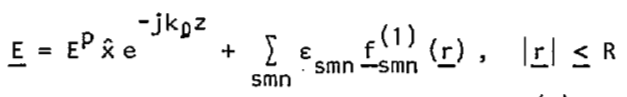

where $R$ is the radius of $S$, and $f_{s m n}^{(1)}$ are the spherical vector wave functions introduced in [4]. The index (1) indicates a standing wave type of function, while an index (4) indicates an outwards propagating type of wave function. Note, that these fields are those that exist with no test antenna in $V$.

Usually, the antenna under test will be ratated in the quasi plane field. This seems to indicate, that some kind of spherical evaluation of the plane wave deviation is desirable.

Field evaluation of plane wave deviation

In each point of the quasi plane wave, the deviation is defined through

$W(\underline{r}) \equiv \frac{\left|\underline{E}^{\varepsilon}(\underline{r})\right|^{2}+n_{0}^{2}\left|\underline{H}^{\varepsilon}(\underline{r})\right|^{2}}{\left|\underline{E}^{\mathrm{P}}\right|^{2}+n_{0}^{2}\left|\underline{H}^{\mathrm{p}}\right|^{2}}$

(CH1672-5/81/0000-000573\$00.75

T981 IEEE.) 
where $n_{0}$ is the free space wave impedance. This figure of merit takes into account both amplitude and phase errors and requires only one scalar for each point. To reduce the number of dimensions, we concentrate on the spherical shape of the plane wave region by introducing the "spherical profile" of the plane wave deviation:

$$
W_{\max }(r) \equiv \max _{|\underline{r}|=r}|10 \log \{w(\underline{r})\}|
$$

Measurement error evaluation of plane wave deviation

We now assume, that $S$ is the minimum sphere enclosing a given test antenna, with radiation described through

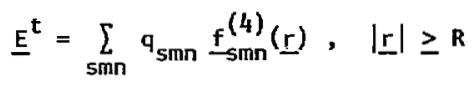

The ideal plane wave has a spherical wave expansion containing only $m= \pm 1$ modes.

Neglecting multiple scattering and assuming generator and load to be matched, Lorentz Theorem may be used to derive the following expression for the measured signal:

$$
u=c \cdot \iint_{S}\left(\underline{E} \times \underline{H}^{t}-\underline{E}^{t} \times \underline{H}\right) \cdot \hat{r} d s
$$

where $\hat{r}$ is the unit vector in the radial direction, and $C$ is a constant. As (5) is linear in the field $(E, H)$, the signal a may be devided into a signal $u_{0}$ corresponding to $\left(E^{P}, H^{P}\right)$ plus an error signal $\Delta u$ corresponding to $\left(\underline{E}, H^{E}\right)$ : $u=u_{0}+\Delta u$. However, this separation is only possible, if the test antenna is
known.

Usually, the a priori knowledge about the antenna is limited to its physical size and some directional properties. Fron this information we know, the effect $P(n)$ radiated in modes of order $n>k_{0} R$ will be very small (except for "supergaín antennas"), [5]. Directive antennas tend to have a relatively high content of higher order modes in the pattern. Further, by rotating the antenna, the power spectrum $\left\{P_{r a d}(n) / P_{\text {rad }}\right\}$ remains unchanged, [6]. These considerations seem to indicate, the power spectrum will be a suitable a priori specification of the test antenna.

Now, for each $n$ redistribute the power on $s$ and $m$ to maximize the measurement error. By doing so the following upper bound $U$ on the relative measurement error is achieved, [7]:

$$
\begin{aligned}
& \left|\frac{\Delta u}{u_{0} \text { i soo }}\right| \leq U \equiv \sum_{n}\left\{\frac{P_{\text {rad }}^{(n)}}{P_{\text {rad }}} \cdot u_{n}^{2}\right\}^{\frac{1}{2}} \\
& u_{n}^{2} \equiv \sum_{s m} \frac{n(n+1)}{2 n+1} \frac{(n+|m|) !}{(n-|m|) !}\left|\frac{\varepsilon_{s m n}}{E_{0}^{g}}\right|^{2}
\end{aligned}
$$

where $u_{0}$ iso is the ideal signal with isotropic radiation and polarization matching. The orthogonality of the wave functions has been exploited. Note, that the numbers $\left\{U_{n}\right\}$ are independent of the test antenna.

The error bound $U$ will be increasingly pessimistic, when the test antenna power pattern spreads out, because the probability for attaining it reduces. This effect can be taken into account by comparing $U$ with the bound that results from measuring with a Hertzian dipole at some reference distance (e.g. the Rayleigh 
distance $8 R^{2}(\lambda)$. For the same reason, the error spectrum $\left\{U_{n}\right\}$ should be compared with the spectrum for a dipole. If $U_{n}$ is small for $n \leq k_{0} R$, then the plane wave deviation will be "small" inside a sphere with radius $R$.

\section{Results}

The error bound formula (6) has been used to determine optimal excitation coefficients for plane cylindrical arrays of Hertzian dipoles.

For this purpose an idealized measuring antenna is used: one dipole on the $z-$ axis plus a number of concentric currentrings in the plane $z=-d$. All currents are $x$-directed. Now, the plane wave deviation inside a sphere of radius $R$ is "minimized" with respect to the ring excitations in the following way. Imagine, that the generated quasi plane wave is used for measurements on a test antenna with a power spectrum, which is constant up to $k_{0} R$ beyond which limit is decreases exponentially. The error bound $U$ for this measurement is minimized with respect to the ring excitations, using nonlinear programining. With $R=14 \lambda, d=240 \lambda$ and a two ring antenna, the solution was found in about 5 seconds of CPU time on an IBM 3033.

Corresponding optimal dipole arrays are abtained by sufficiently close sampling of each ring. The sampling requirements are studied in [7].

Fig. 1 shows error spectra $\left\{U_{n}\right\}$ for a couple of optimized $r i n g$ antennas. Radius of the plane wave region is $R=10 \lambda, k_{0} R \simeq 63$ in this case.

Fig. 2 shows spherical profiles for a couple of sampled optimal ring antennas. The measurement distance is about one third of the Rayleigh distance. It was found, that the effect of the increased ring radi $i$ on the profile was negligible. But a significant reduction in the sensitivity to small weight errors was obtained. Replacing the dipoles with open circular waveguides (radius $0.35 \lambda$ ) did not lead to visible changes in the spherical profiles.

\section{Acknowledgements}

The auther wishes to thank Jesper E. Hansen, F. Holm Larsen and A.C. Ludwig for many discussions on the subject.

\section{References}

1. J. E. Hansen, "Spherical Near-Field Testing of Space-craft Antennas", ESA Journal 1980, Vol. 4, pp. 89-102.

2. A.C. Ludwig and F. Holm Larsen, "Spherical near-field measurements from a 'compact-range' viewpoint", IEEE International Conference on Antennas and Propagation, York, April 1981, Proceedings.

3. J.C. Bennett and E.P. Schoessow, "Antenna near-field/far-field transformation using a plane wave synthesis technique"', Proc. IEEE, 125, No. 3, 1978, pp. $179-184$.

4. F. Jensen, "Electromagnetic near-field far-field correlations", Ph.D.-dissertation (LD15), Electromagnetics Institute, Tech. Univ. of Denmark, 1970.

5. J.A. Stratton, "Electromagnetic Theory", McGraw-Hill, Chapter VII, 1941.

6. R.F. Harrington, "On the Gain and Beamwidth of Directional Antennas", IRE Trans. on Antennas and propagation, July, 1958, Pp. 219-225.

7. J. Hald, "Numerical optimization of near-field antenna arrays", Ph.D.-dissertation, Electromagnetics Institute, Tech. Univ. of Denmark, 1981. 


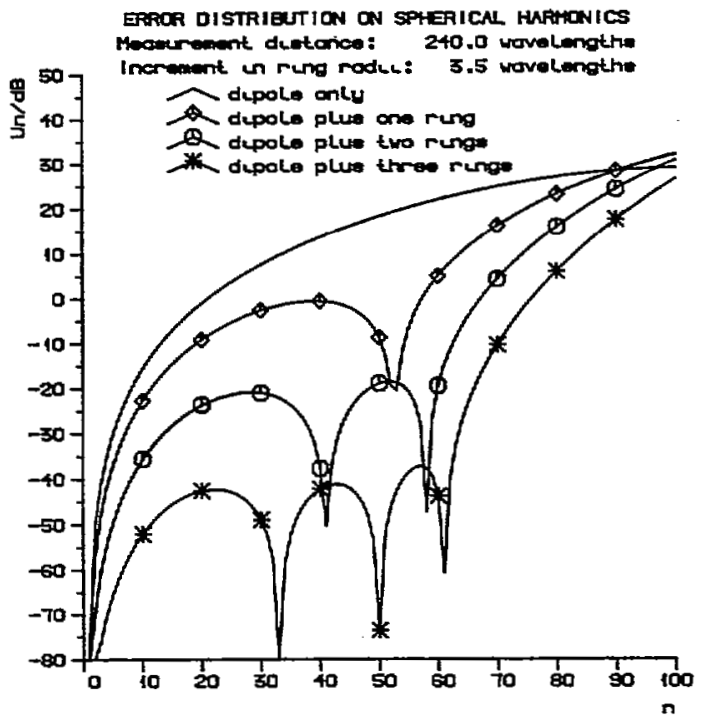

Fig. 1. Error spectra $\left\{U_{n}\right\}$ for a dipole and for three optimal ring antennas.

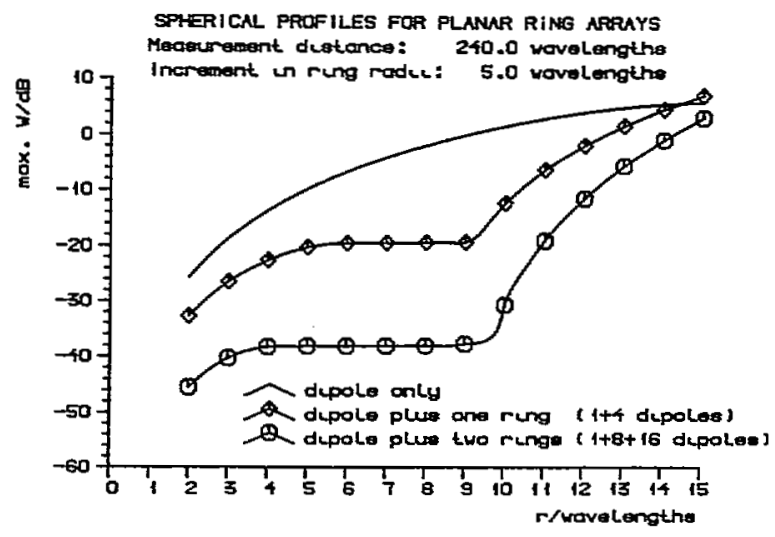

Fig. 2. Field evaluation of two optimal plane cylindrical dipole arrays. 
SESSTON 20

LOW FREQUETYCY ANTIENNAS

THURSDAY 1:30 - 5:00

SANTA BARBARA, ROOM C

Chairman: C. A. Balanis

University of West Virginia

Norgantown, WV

Page

1. A Frequency Tracking, Tuned, Receiving

Monopole

Gerome Reeve and Arthur Wainwright, National Bureau of Standerds, Boulder, CO

2. A Compact Wideband HF Transmitting

Antenna

Richard K. Royce, Neval Research

Laboratory, Washington, DC

3. Broadband Trapped Multiple-Wire Antennas

W.A. Edson, SRI International, Menlo Park, CA

4. Analysis of a V-Loop Antenna

Naami Amano, Sony Corporation, Tokyo, Japan and Risaburo Sato, Tohoku University,

Sendai, Japan

5. Decoupling of a Honopole Antenna with a

Radial-Transmission-Line Choke

T.G. Dalby, Boeing Military Airplane

Company, Seattle, WA

6. Monopole Antenna Patterns on Finjte Size

Composite Ground Plones

Constantine A. Balenis, West Virginia

University, Morgantown, $W V$ and Dennis

DeCarlo, Naval Air Test Center, Patuxent

River, MS 


\title{
A FREQUENCY TRACKING, TUNED, RECEIVING MONOPOLE
}

\author{
Gerome Reeve and Arthur Wainwright \\ Electromagnetic Fields Division \\ National Bureau of Standards \\ Boulder, Colorado 80303
}

\begin{abstract}
Various active antennas have been utilized for receiving purposes in the low to medium high frequency region because of their advantage of high sensitivity for a given size. They have certain drawbacks however, such as limited dynamic range and intermodulation distortion. While no technical approach is satisfactory for all purposes, the antenna described in this paper was designed for weak short wave signal reception in the presence of very large out-ofband signals, e.g., commercial broadcast stations.
\end{abstract}

The antenna proper consists of a 2.5 meter collapsible monopole (whip) which is resonated with an inductance, and a pair of matched voltage variable capacitive diodes, to the frequency of interest. The signal at the base of the antenna is then applied to a V-MOS power FET follower circuit with negative feedback yielding an output at a $50 \Omega$ impedance. Octave bands can be tuned with the voltage variable capacitive diodes and are set to match the bands of a typical field intensity meter (FIM). Operating bandwidths vary from approximately $10 \mathrm{kHz}$ at $250 \mathrm{kHz}$ to $1 \mathrm{MHz}$ at $30 \mathrm{MHz}$.

Referring to figure 1 , a simplified block diagram, we see that the tuning of the antenna is controlled by the FIM. An output voltage available at the rear of the FIM is proportional to the frequency to which it is tuned. This voltage is digitized in an A/D converter and used as the address for a ROM lookup table. The output of the ROM memory goes to a D/A converter and tunes the variable capacitive diode circuit to the proper frequency. A front panel control allows manual tuning if desired. Band switching signals, also available from the FIM, activate reed relays to switch the resonating inductors in the antenna circuit. Broadband untuned operation of the antenna is a1so available when desired, or to be used below $250 \mathrm{kHz}$, which is the lowest octave band available in the FIM being used. A $20 \mathrm{~dB}$ capacitive divider switchable attenuator a llows signals of up to 7 volt/meter to be handled in the untuned configuration with intermodulation products $>80 \mathrm{~dB}$ below signal level. The data shown in the following figures however is with the attenuator in the zero $\mathrm{dB}$ position.

Figure 2 shows the response of the antenna in terms of antenna factor (AF) which is:

$$
A F=20 \log _{10} \frac{E \text { field }}{V \text { out }} .
$$

There are several ways to represent the sensitivity of antennas, not all of which are equally meaningful to the person making field intensity measurements. In figure 3 the noise floor of the antenna

U. S. Government work not protected by U. S. copyright. 
is plotted in terms of the equivalent RMS field strength in $d B$ $\mu$ volts/meter which that noise represents. Also plotted on the same curve is the quasi-minimum atmospheric background noise level (ECAC-CR-76-074 October 1976) which is useful in gauging the ability to measure background noise levels in typical locations.

Figure 4 shows second and third order intermadulation responses versus signal level for interfering signals located within the octave below the frequencies being tuned. Figures 5 and 6 show the dramatic improvement in spectrum analyzer performance using this antenna in the tuned mode. In figure 5 the antenna is operating in the broadband configuration with the resulting large number of distartion products, many generated with in the spectrum analyzer itself. Figure 6 shows the $4-8 \mathrm{MHz}$ frequency band tracked with the spectrum analyzer in this range.

The antenna is mounted on a small aluminum enclosure which connects to a control/interface unit. The ROM lookup table and analog conditioning circuits are located on a plug-in personality card so the tuning function can be adapted to other FIMs. Internal batteries allow for portable operation. For more versatility an IEEE 488 bus interface is being contemplated.

It is felt that this antenna combines flexible performance with enhanced sensitivity accompanied by low distortion products, in the tuned mode of operation.

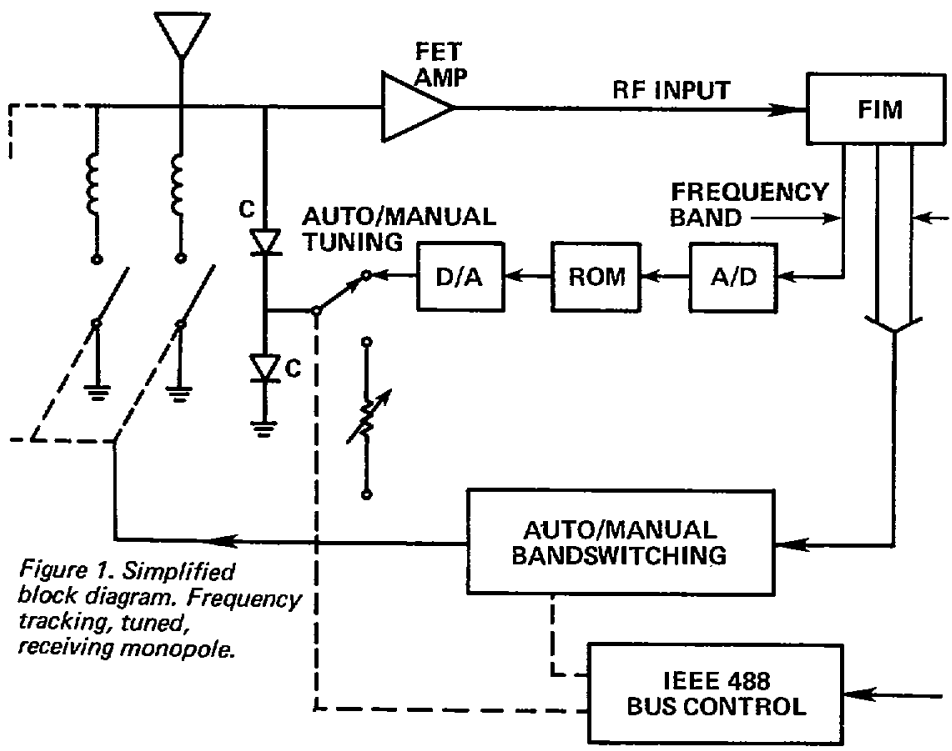




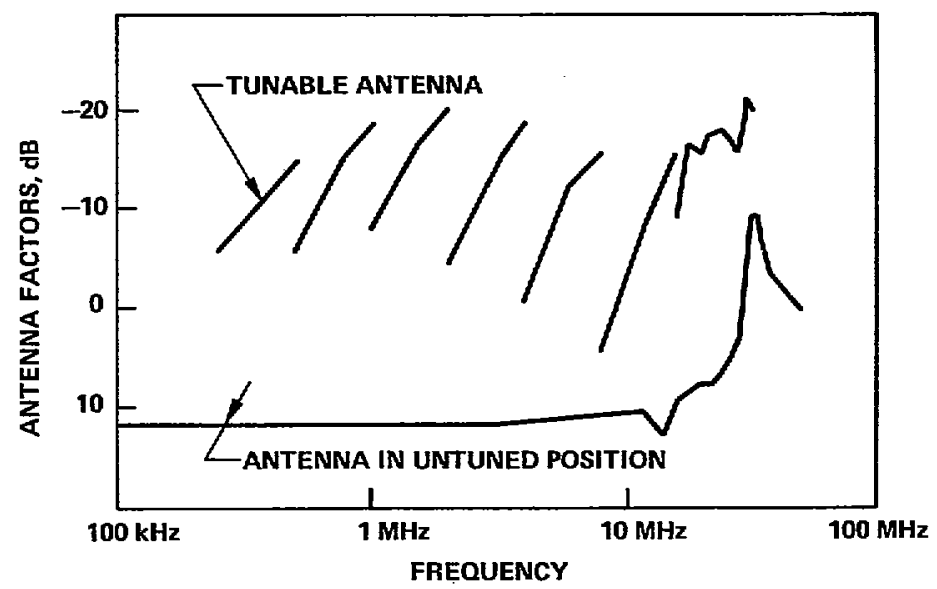

Figure 2. Antenna factors.

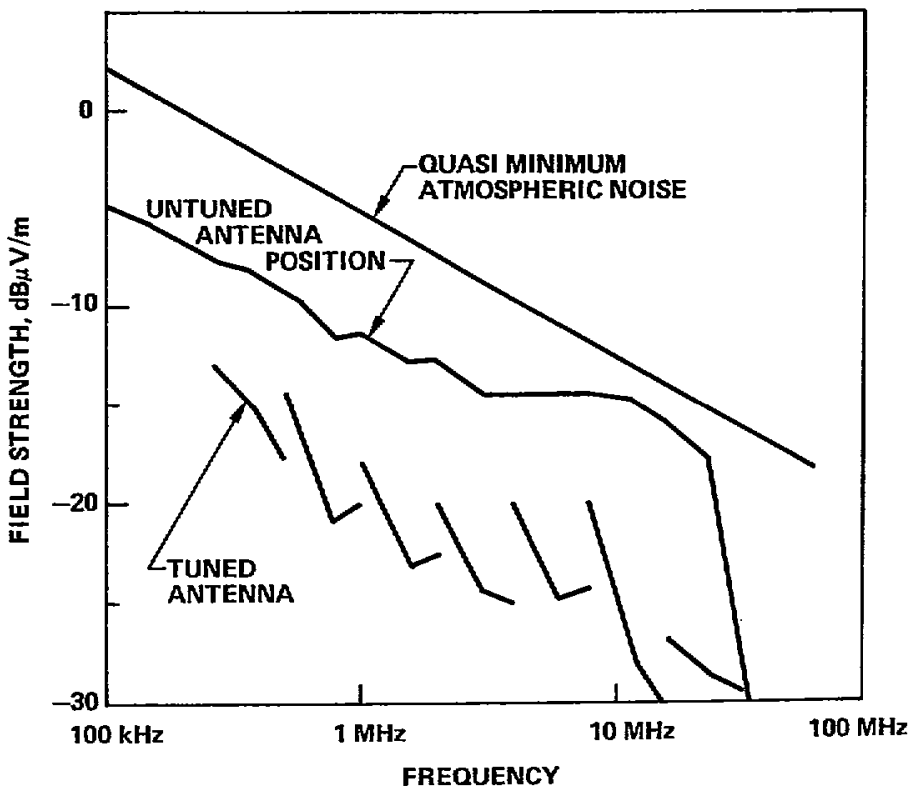

Figure 3. Sensitivity (noise floor) of antenna. 